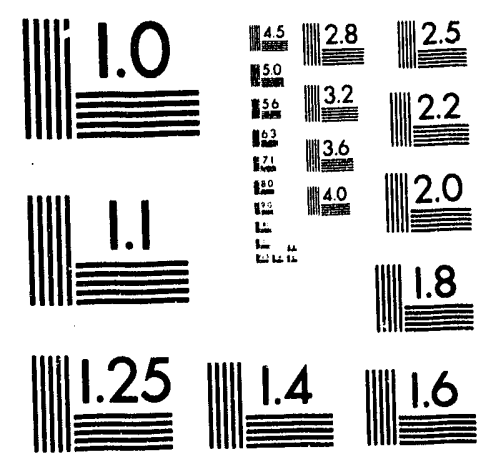



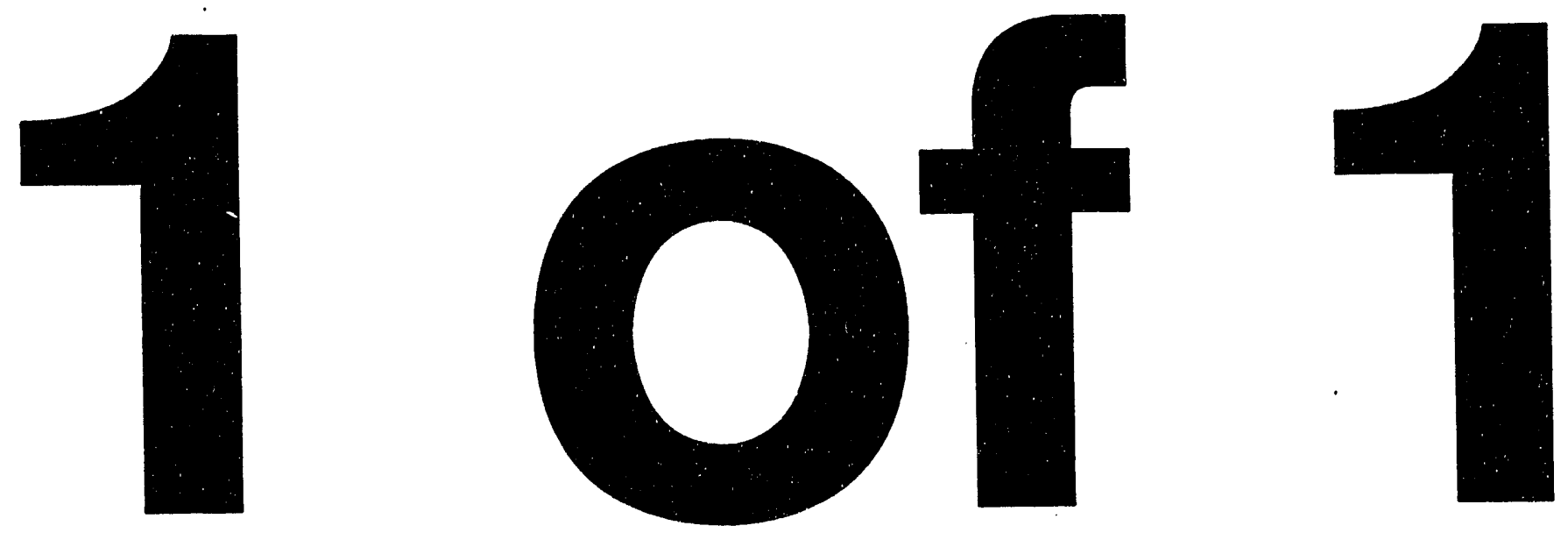
LBL-34374

\title{
DIFFRACTION AND HOLOGRAPHY OF PHOTOELECTRONS AND FLUORESCENT X-RAYS
}

\author{
Charles S. Fadley \\ Materials Sciences Division \\ Lawrence Berkeley Laboratory \\ University of California \\ Berkeley, California 94720 \\ and \\ University of California at Davis \\ Department of Physics \\ Davis, California 95616
}

This work was supported by the Director, Office of Energy Research, Office of Basic Energy Science, Materials Sciences Division of the U.S. Departmerit of Energy under Contract Number DE-AC03-76SF00098. 
Invited paper to appear in the

Proceedings of the Spring 1993 Meeting of the Materials Research Society

\title{
DIFFRACTION AND HOL.OGRAPHY OF PHOTOELECTRONS AND FLUORESCENT X-RAYS ${ }^{+}$
}

\author{
CHARLES S. FADLEY
}

Department of Physics, University of California-Davis, Davis, CA 95616

Materials Sciences Division, Lawrence Berkeley Laboratory, Berkeley, CA 94720

\section{ABSTRACT}

Photoelectron diffraction is by now a powerful technique for studying surface structures, with special capabilities for resolving chemical and magnetic states of atoms and deriving direct structural information from both forward scattering and backscattering. Fitting experiment to theory can lead to structural accuracies in the 0.03 range. Holographic inversions of such diffraction data also show considerable promise for deriving local three-dimensional structures around a given emitter with accuracies of $0.2-0.3 \AA$. Resolving the photoelectron spin in some way and using circularly polarized radiation for excitation provide added dimensions for the study of magnetic systems and chiral experimental geometries. Synchrotron radiation with the highest brightness and energy resolution, as well as variable polarization, is crucial to the full exploitation of these techniques. X-ray fluorescence holography also has promise for structural studies, but will require intense excitation sources and multichannel detection to be feasible.

\section{INTRODUCTION}

Photoelectrons emitted from core levels represent localized sources of outgoing waves which can then scatter from nearby atoms to produce diffraction patterns. This paper deals largely with the ways in which such diffraction patterns can be used to determine atomic, as well as magnetic, structures in materials [1-3]. The analysis of such data in a more recently suggested holographic manner so as to directly image atoms [4,5] is also considered. The benefits that synchrotron radiation brings to such studies are emphasized. Core-level fluorescent x-rays also should in principle lead to such diffraction patterns, and the potential for using these patterns for holographic imaging is also discussed.

\section{PHOTOELECTRON DIFFRACTION}

Photoelectron diffraction patterns are by now well known and much studied, and have lead to the increasing use of this technique for surface structure studies $[1,2,3]$. The fundamental measurement is illustrated in Fig. 1. A photoelectron is emitted from a core level, and its intensity is measured as a function of its direction or its energy above a singlecrystal sample, yielding what can be termed scanned-angle or scanned-energy data, respectively. In terms of the electron wave vector k, this is equivalent to measuring intensity as a function of direction $\hat{k}=\underline{k} /|\underline{k}|$ or magnitude $k=|\underline{k}|$. Intensity variations are produced by the interference of the unscattered or direct wave component $\phi_{0}$ and the various scatteredwave components $\phi_{j}$.

The resulting photoelectron intensity as a function of wave vector can be written in a simple single scattering picture as [1]:

$$
\begin{aligned}
I(k) & \propto\left|\phi_{0}+\Sigma_{j} \phi_{j}\right|^{2} \\
& \propto\left|\phi_{0}\right|^{2}+\Sigma_{j}\left(\phi_{0}{ }^{*} \phi_{j}+\phi_{o} \phi_{j}{ }^{*}\right)+\Sigma_{j} \Sigma_{k} \phi_{j} \phi_{k}{ }^{*},
\end{aligned}
$$

where $\phi_{j}$ and $\phi_{k}$ are arbitrary scattered waves. For the illustrative case of photoelectron emission from an s subshell into an outgoing $\phi_{0}$ with $p$ 
character, the individual wave components here can be written out more explicitly in terms of (cf. Fig. 1): dipole matrix elements that are for linearly polarized radiation proportional to the pclarization direction (E) dotted into the relevant emission direction $\left(\hat{k}\right.$ or $\left.\underline{r}_{j} / \mathbf{r}_{j}=\hat{\mathbf{r}}_{j}\right)$; inelastic exponential decay factors exp $\left(-L / 2 \Lambda_{e}\right)$, with $L$ equal to the total length for some path below the surface and $\Lambda_{e}$ the inelastic attenuation length; scattering factors $f_{j}\left(\theta_{j}\right)$ involving both an amplitude $\left|f_{j}\left(\theta_{j}\right)\right|$ and a phase shift $\Psi_{j}\left(\theta_{j}\right)$ that are functions of the scattering angle $\theta_{j} ;$ Debyewaller factors $W_{j}$ that allow for attenuation of interference due to vibrational effects; and finally, phase shifts due to path length differences of the form $\exp \left[i k r_{j}\right] \exp \left[-i \underline{k} \cdot \underline{r}_{j}\right]=\exp \left[i k r_{j}\left(1-\cos \theta_{j}\right)\right] \cdot A l 1$ structural information is thus contained in these last factors, with the path length difference between $\phi_{0}$ and $\phi_{j}$ being given by $r_{j}\left(1-\cos \theta_{j}\right) \cdot$ Eq. 1 then becomes:

$$
\begin{gathered}
I(\underline{k}) \propto\left|(\hat{\epsilon} \cdot \hat{k}) \exp \left(-L_{o} / 2 \Lambda_{e}\right)+\Sigma_{j}\left(\hat{\epsilon} \cdot \hat{r}_{j} / x_{j}\right)\right| \underline{f}_{j}\left(\theta_{j}\right) \mid w_{j} \exp \left(-L_{j} / 2 \Lambda_{e}\right) \\
\quad \times\left.\exp \left[i\left\{k r_{j}\left(1-\cos \theta_{j}\right)+\Psi_{j}\left(\theta_{j}\right)\right\}\right]\right|^{2},
\end{gathered}
$$

or, in more convenient notation:

$$
I(\underline{k}) \propto\left|F_{0}+\Sigma_{j} F_{j} \exp \left[-i \underline{k} \cdot \underline{r}_{j}\right]\right|^{2},
$$

with

$$
\begin{aligned}
& F_{0}=(\hat{\epsilon} \cdot \hat{k}) \exp \left(-L_{o} / 2 \Lambda_{e}\right) \\
& F_{j}=\left(\hat{\epsilon} \cdot \hat{r}_{j} / r_{j}\right)\left|f_{j}\left(\theta_{j}\right)\right| w_{j} \exp \left(-L_{j} / 2 \Lambda_{e}\right) \exp \left[i k r_{j}\right] \exp \left[i \Psi_{j}\left(\theta_{j}\right)\right] .
\end{aligned}
$$

Here, one portion of the phase factor due to path length (exp[ikr $j$ ) is now incorporated into the $F_{j} \cdot s$. Eq. 3 can also be formally generalized to include multiple scattering [5a,6], in which case each $F_{j}$ must include a sum over the various single and multiple scattering pathways m with different total lengths $\mathrm{L}_{\mathrm{mj}}$ that terminate in scatterer $j$ just before going to the detector; within each multiple-scattering pathway, there also will be products of successive path-length phase factors and scattering factors. For emission from a subshell other than $s$ (i.e. linitial $>0$ ) the above expressions become more complex due to sums over initial and final magnetic quantum numbers and final-state interference between the $l_{\mathrm{final}}=1+1$ and 1-1 channels allowed by the dipole selection rules $[6 a, 7]$.

Expanding the square in Eq. 3 now yields

$$
\begin{aligned}
I(\underline{k}) & \propto\left|F_{o}\right|^{2}+\Sigma_{j}\left[F_{o}{ }^{*} F_{j} \exp \left\{-i \underline{k} \cdot \underline{r}_{j}\right\}+F_{o} F_{j}{ }^{\star} \exp \left\{i \underline{k} \cdot \underline{r}_{j}\right\}\right] \\
& +\Sigma_{j} \Sigma_{k}\left\{F_{j}{ }^{\star} F_{k} \exp \left\{i \underline{k} \cdot\left(\underline{r}_{j}-\underline{r}_{k}\right)\right\}+F_{j} F_{k}{ }^{\star} \exp \left\{-i \underline{k} \cdot\left(\underline{r}_{j}-\underline{r}_{k}\right)\right\}\right\} .
\end{aligned}
$$

$\left|F_{o}\right|^{2} \equiv I_{0}(\underline{k})$ is just the intensity in the absence of any scattering. A normalized intensity function $\chi(\underline{\mathrm{k}})$ can now be calculated, very much as in the analysis of extended $x$-ray absorption fine structure (ExAFS):

$$
\chi(\underline{k})=\left[I(\underline{k})-I_{0}(\underline{k})\right] / I_{0}(\underline{k})^{1 / 2} \text {. }
$$

and this yields

$$
\begin{gathered}
\chi(\underline{k}) \propto\left(\left|F_{o}\right|\right)^{-1} \Sigma_{j}\left[F_{o}(\underline{k})^{*} F_{j}(\underline{k}) \exp \left\{-i \underline{k} \cdot \underline{r}_{j}\right\}+F_{o}(\underline{k}) F_{j}(\underline{k})^{*} \exp \left\{i \underline{k} \cdot \underline{r}_{j}\right\}\right] \\
+\left(\left|F_{o}\right|\right)^{-1} \Sigma_{j} \Sigma_{k}\left[F_{j}(\underline{k})^{*} F_{k}(\underline{k}) \exp \left\{i \underline{k} \cdot\left(\underline{r}_{j}-\underline{r}_{k}\right)\right\}+F_{j}(\underline{k}) F_{k}(\underline{k})^{*} \exp \left\{-i \underline{k} \cdot\left(\underline{r}_{j}-\underline{r}_{k}\right)\right\}\right] \cdot
\end{gathered}
$$




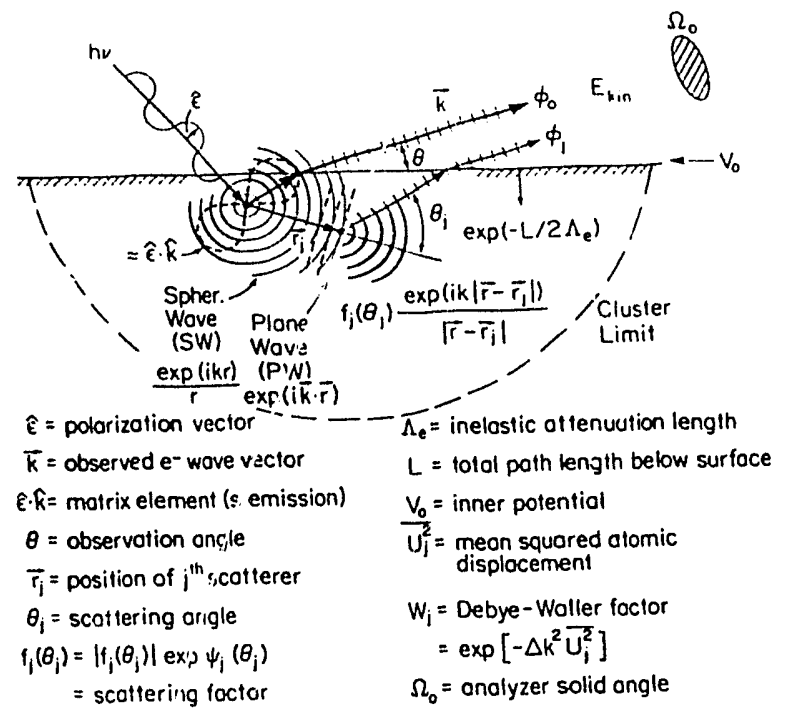

Fig. . - The basic process involved in photoelectron diffraction, with important physical variables indicated. Only single scattering is indicated for simplicity. In a holographic interpretation of such measurements, the direct or unscattered wave $\phi_{0}$ is identified with the reference wave, and the scattered waves $\phi_{j}$ are identified with object(subject) waves.

Fig. $2-$ The geometry of $c(2 \times 2) s / N i(001)$ is shown together with Fourier transform holographic images from Eq. 10, as based upon s 2p emission at $1327 \mathrm{eV}$. The hologram analyzed has cylindrical symmetry about the $z$ axis, and extends from $10^{\circ}$ to $50^{\circ}$ above the surface. Images are shown in both the $x y(=s)$ and $x z$ planes. No scattered-wave correction has been made, and results are shown for both experiment ( $(a)$ and (c)) and single-scattering theory (b) and (d)). The positions of nearestneighbor $(N-N)$ and next-nearestneighbor $(N-N-N)$ s atoms are indicated. The vertical dashed line indicates the known positions of these atoms. [From Thevuthasan et al. ref. 15c]

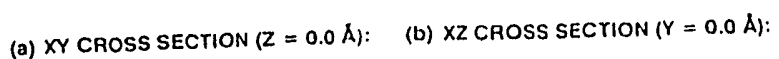
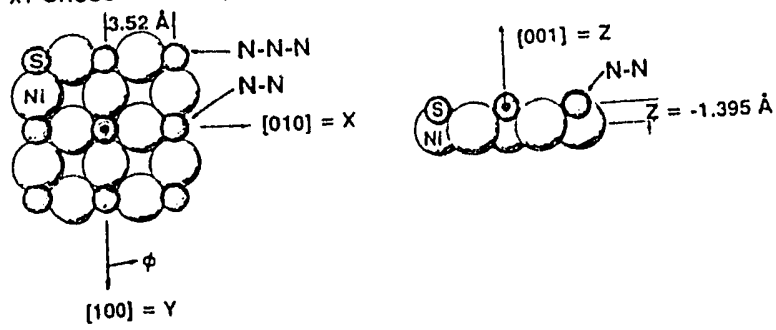

(a) $|F T|, X Y$ PLANE, EXPERIMENT:
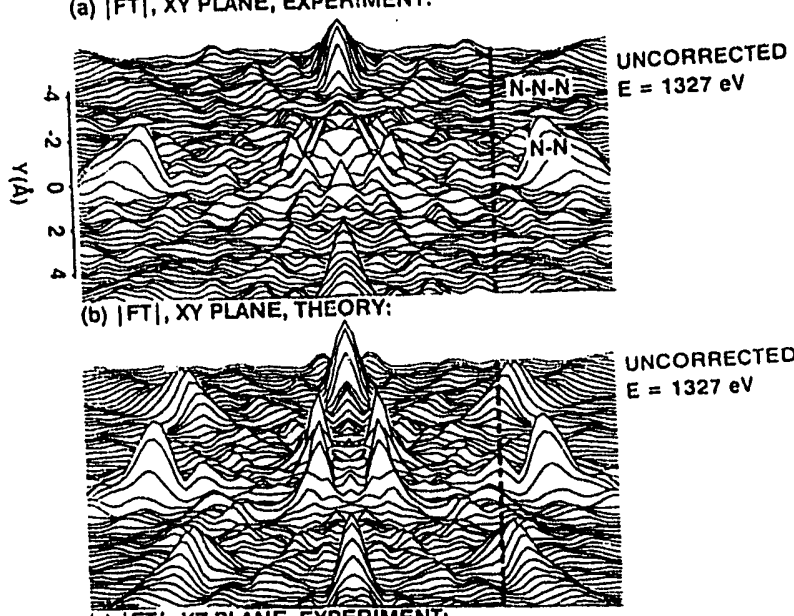
(c) $|F T|, X Z$ PLANE, EXPERIMENT:

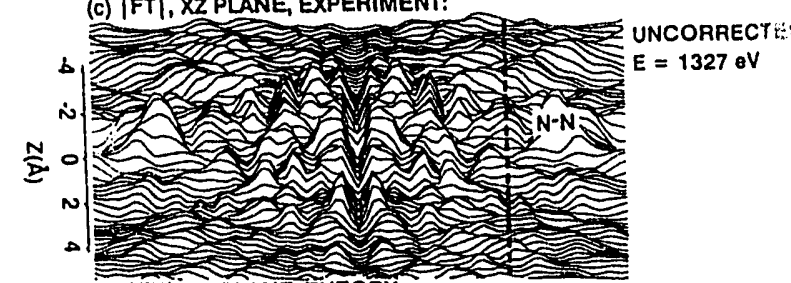

(d) |FTI, XZ PLANE, THEORY:

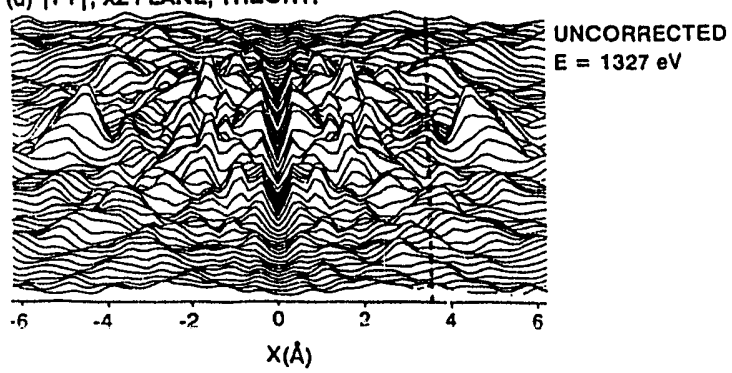


This form is useful in considering holographic analyses of diffraction. One common approximation is to assume that the scattered waves $\phi_{j}$ and $\phi_{k}$ are small in amplitude with respect to $\phi_{O^{\prime}}$ so that the cross terms $\phi_{O}{ }^{*} \phi_{j}$ and $\phi_{O} \phi_{j}{ }^{\star}$ in Eq. 1 dominate the structural information. This directly leads via Eqs. 3 and 6 to

$$
\begin{array}{r}
\chi(\underline{k}) \propto 2 \Sigma_{j}\left(\hat{\epsilon} \cdot \hat{r}_{j} / r_{j}\right)\left|f_{j}\left(\theta_{j}\right)\right| w_{j} \exp \left(-L_{j} / \Lambda_{e}\right) \\
\times \cos \left[k r_{j}\left(1-\cos \theta_{j}\right)+\Psi_{j}\left(\theta_{j}\right)\right] .
\end{array}
$$

This form directly shows that Fourier transforms of scanned-energy data over some interval $\Delta k$

$$
F_{\Delta k} \propto \int_{\Delta k} \chi(\underline{k}) \exp [-i k r] d k ，
$$

should be useful for deriving path length differences $r_{j}\left(1-\cos \theta_{j}\right)$, as discussed in a number of prior studies $[6 \mathrm{~b}, 8]$.

Various types of information can thus be derived from such photoelectron diffraction patterns, and their primary characteristics are summarized below. More detailed discussions with illustrative examples appear elsewhere $[1-3]$.

-Atom specificity: The measurements are inherently atom-specific, since core level energies can always be found that are unique to a given atom. Thus, the local structure around each of the atomic types in a sample can be studied.

-Chemical-state specificity: For many systems, core levels furthermore exhibit chemical shifts or surface shifts, so that the structure around different chemical/surface states of the same atom can in principle be studied separately. This has been applied for example to distinguishing surface and bulk metal atoms [9a], the different sites in adsorbed molecules [9b] or atoms [9c], and different layers near epitaxial interfaces [9d]. This application requires energy resolutions in the $0.1-0.3 \mathrm{eV}$ range, and is thus well-suited to synchrotron radiation studies.

-Spin specificity: In atoms with a net magnetic moment, there can also be exchange-produced splittings of core levels in which the spin-up and spindown photoelectrons are separated in energy [10]. An external spin detector can also be used to directly measure the spin polarization $P=$ $[I(t)-I(t)] /[I(t)+I(t)]$ over a core spectrum [11]. This suggests the use of spin polarized photoelectron diffraction in the study of magnetic materials, as amplified below. In order to enhance magnetic scattering effects, kinetic energies of approximately $100 \mathrm{eV}$ are required, thus again in general implying synchrotron radiation for excitation. In addition, circularly polarized radiation can be used in excitation of spin-orbitsplit levels to enhance one or the other outgoing spin polarization; this again requires synchrotron radiation, as discussed further below.

-Simple forward scattering: In measurements at photoelectron kinetic energies of about 500 ev or higher, the scattering amplitude $\left|f_{j}\left(\theta_{j}\right)\right| i s$ highly peaked in the forward direction ( $i . e$. near $\theta_{j}=0$ ). Various studies have by now shown that such forward scattering or forward focussing peaks can be directly used to determine bond directions for adsorbed molecules $[1,3]$ and low-index directions for single crystals and epitaxial overlayers $[1,2]$. The higher kinetic energies required for this kind of measurement have led to its being performed primarily with laboratory $x-r a y$ sources in the 1.2-1.5 kev range, but higher brightness synchrotron radiation sources in the 500-1500 eV range would be equallÿ useful for this work. 
-Back scattering: In measurements at lower photcelectron kinetic energies of less than about $300 \mathrm{ev}$, there is also a significant degree of back scattering, and this can be used in several ways to extract structural information concerning atoms that are "bekind" the emitter as viewed by the detector $[1,6 b, 12,13]$. Synchrotron radiation is again necessary to insure sufficiently low kinetic energies.

-Path-length differences: Another direct form of structural information that can be obtained is the path length difference associated with a given scatterer $j: r_{j}\left(1-\cos \theta_{j}\right)[6 b, 8,12,13]$. As noted above, this requires Fourier transforming scanned-energy data over some interval $\Delta k$, and in turn requires synchrotron radiation to vary energy (and thus $k$ ) in small steps.

-Accurate surface structures: In a smallex, but growing, number of studies to date, it has been found possible also to determine more detailed surface structures by fitting experimental diffraction patterns of either the scanned-angle or scanned-energy type to theoretical simulations for various possible trial geometries $[1,2 a, 12]$. Theoretical calculations have been carried out at both the single scattering $[1,2 a]$ and more accurate multiple scattexing $[1,6,12]$. With careful analysis of such fits, e.g., via R factors, accuracies in the approximately $0.03 \AA$ range have been obtained. However, further work is needed to speed up such structure searches and the multiple scattering calculations needed for the highest ultimate accuracy. Finally, more rapid data acquisition methods are also called for; these will benefit from rext-generation higher-brightness synchrotron sources ns well.

\section{PHOTOELECTRON HOLOGRAPHY}

More recently, it has been suggested by szöke [4] that such photoelectron diffraction patterns can be treated as holograms, with the unscattered wave $\phi_{0}$ being identified as the reference wave of the hologram, and the scattered waves $\phi_{j}$ being identified as the object waves. A diffraction pattern that is somehow measured over a relatively large number of points in $\underline{k}$ space is then tranformed into a direct three-dimensional image of the atoms surrounding a given atom using a Fourier-transform-like integral. This holographic interpretation of diffraction data is in a much more developmental stage, but several encouraging experimental studies have been carried out to date $[14-17]$.

The hologram is now just the intensity $I(\underline{k})$, or more conveniently the normalized function $\chi(\underline{k})$, as measured over a range of solid angles and/or energies. The simplest imaging procedure, as first suggested by Barton [5], makes use of the Helmholtz-Rirchoff theorem from optics to calculate the atomic image $U(\underline{r})$ (actually the source wavefield) associated with the hologram from:

$$
u(x, y, z) \propto\left|\iint_{S} \chi(\underline{k}) \exp [i \underline{k} \cdot \underline{\underline{r}}] \mathbf{d} \sigma_{k}\right|,
$$

where the integral on the direction of $\underline{k}$ is over the spherical surface on which the hologram is measured. Note that $\chi(k)$ has here been multiplied by the complex conjugate of the direction-dependent part of the phase factor due to path length difference exp[-ikㅏㅁㅣ], and that the magnitude of $\underline{k}$ is fixed. Applying Eq. 9 to $\chi(\underline{k})$ as written in Eq. 6 them immediately predicts the existence of both real and twin images at $\pm r_{j}$, as well as weaker self-interference images at $\pm\left(\underline{r}_{j}-\underline{r}_{k}\right)$, both potentially complicating features in structural studies. Self-interference effects have been predicted to be generally $\leq 10-20 \%$ as strong as the real/twin images, 
although they may not always be negligible [18b]. Further taking the $z$ axis to be along the symmetry axis of the hologram and thus usually also to be perpendicular to the surface and then projecting $\chi(k)$ onto the $k_{x}, k_{y}$ surface plane permits doing a two-dimensional Fourier transform with $z$ as a variable parameter to yield the image $U$ in a given $z$ plane as [5]:

$$
U(x, y, z) \propto\left|\iint\left\{x(\underline{k}) \exp \left[i k_{z} z\right]\right\} \exp \left[i\left(k_{x} x+k_{y} y\right)\right] d k_{x} d k_{y}\right| .
$$

If the full opening angle of the hologram as centered on the z-axis normal to the surface is defined to be $\alpha$, it can further be shown [5] that the uncertainties with which positions can be determined in the three coordinates are given by: $\Delta x=\Delta y=1.22 \pi /[\mathrm{ksin}(\alpha / 2)]=0.61 \lambda_{\mathrm{e}} / \sin (\alpha / 2)$ in

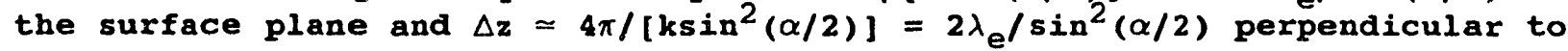
the surface plane, where $\lambda_{e}$ is the electron de Broglie wavelength. These uncertainties can also be inversely related via the Uncertainty Principle to the ranges $\Delta k_{x}, \Delta k_{y}$, and $\Delta k_{z}$ that are spanned by the hologram [19].

Holographic images may also be distorted due to anisotropy in the amplitudes of both the reference wave $\phi_{o}$ and the scattered waves $\phi_{j}$, as well as the often significant phase shifts $\Psi_{j}$ due to scattering. Possible solutions to these problems are to eliminate or correct regions of the hologram that are most non-ideal, as for example, over the forward scattering peaks [18]. As one example of this, we show in Fig. 2 experimental and theoretical images for the well-defined test case of $c(2 \times 2) S$ on $\mathrm{Ni}(001)$ obtained by Thevuthasan et al. [15c] in two different symmetry planes, as shown in the atomic geometry. Here, data for $S 2 p$ emission at $1327 \mathrm{eV}$ have been obtained for takeoff angles greater than $10^{\circ}$ above the surface in order to avoid strong forward scattering and multiple scattering effects that may occur for emission directions more nearly parallel to the surface. The images of the nearest-neighbor sulfur atoms are clear in both experiment and theory, and there is good agreement as to the degree of shift relative to the true positions of about $1.0 \AA$.

To improve the image positions, it is necessary to somehow correct for anisotropic scattering amplitudes and/or phase shifts in doing the imageproducing transform [20]. One correction method proposed by Tonner, saldin and co-workers [20a] is simply to normalize $\chi(\underline{k})$ by a generalized scattered-wave strength $F_{j}$ during the integration, which yields a new image function $U$ :

$$
U^{\prime}(x, y, z) \propto l \iint\left\{x(\underline{k}) \exp \left[i k_{z} z\right] / F_{j}(\underline{k}, \underline{\underline{x}})\right\} \exp \left[i\left(k_{x} x+k_{y} y\right)\right] d k_{x} d k_{y} \mid
$$

This has been termed the scattered-wave-included Fourier transform (SWIFT) method. In practice, this procedure has to date involved simply dividing by a plane-wave or spherical-wave scattering factor, which may then have to be adjusted with position in space so as to allow for the different types of scatterers present $[15,20]$. The latter adjustment thus requires some advance knowledge of the structure, or an iterative approach. $F_{j}$ also can in principle allow for the anisotropy in the outgoing reference wave, as noted above.

The overlap of real and twin images is a problem shared with optical holography, but it is potentially more serious in images of surface structures, since the surface inherently breaks the inversion symmetry along its normal, and thus the twins of substrate atoms may overlap the regions in space occupied by adsorbate or overlayer atoms. One solution to this problem is to note that, for some cases, the region of the hologram 
most strongly affected by some atom at $\underline{\underline{r}}$ is well localized in a sclic.ari:a region centered on $\underline{x}_{\text {; }}$ this was first demonstrated in theoretical simulations by saldin et al. [21]. Analyzing only this portion of the hologram then may lead to an image in which the twin from another atom at $\underline{\underline{r}}$ is suppressed, as suggested by saiki et al. for scanned-angle data from cases dominated by forward scattering [22]. For backscattering cases at lower energies, Tong et al. [20b] have proposed analyzing scanned-energy data over only small windows in direction in order to emphasize a single scatterer behind the emitter.

In Fig. 3, we show the effects of simultaneously using these last two image improvement procedures, again for the case of $c(2 \times 2) s / N i(001)$ [15c]. only the right half of the hologram has been analyzed to focus on the position of the nearest neighbor along $+x$, and the sWIFT procedure has been applied in doing the image formation. The agreement between experiment and theory is again excellent, and the peak positions have improved to within about $0.3 \AA$ of the known structure. This example thus suggests that even single-energy holographic images for adsorbate overlayers or thin epitaxial layers can be obtained with sufficient accuracy to be used for ruling out many possible structures and providing excellent starting points for more accurate final trial-and-error refinements. other single-energy, SwIFTcorrected results presented in this symposium for bulk Cosi 2 at 700 eV are also encouraging [16a]. However, previous work on bulk specimens of Cu [14a] and si [15a] at a single energy suggest that the presence of inequivalent emitters in several layers can lead to strong image distortions along forward scattering directions.

A more general approach for suppressing twins, as well as other deleterious effects in images, has also been suggested by Baxton [5b], and it involves making phased summations of transforms obtained at different energies $E_{i}$ with wave vectors $k_{i}$ according to:

$$
u^{\prime \prime}(x, y, z) \propto\left|\Sigma_{i} \exp \left[-i k_{i} r\right] \iint_{S} \chi\left(\underline{k}_{i}\right) \exp \left[i \underline{k}_{i} \cdot \underline{r}\right] d \sigma_{k}\right|
$$

or

$$
\begin{gathered}
\propto \mid \Sigma_{i} \exp \left[-i k_{i} r\right] \iint\left\{x\left(\underline{k}_{i}\right) \exp \left[i k_{i z} z\right] / F_{j}\left(\underline{k}_{i}, \underline{x}\right)\right\} \\
\cdot \exp \left[i\left(k_{i x} x+k_{i y} y\right)\right] d k_{x} d k_{y} \mid .
\end{gathered}
$$

This sum can in principle be performed either with or without correction for the scattered wave, although it has been included above in dividing again by $F_{j}(\underline{k}, \underline{r})$ in Eq. 12b. In doing this sum, we have multiplied by the conjugate of the remaining phase factor due to path length difference exp[ikr], with $\chi(k)$ containing such factors inside of the $F_{j} s$ (cf. Eqs. 2 and 3 ). The sum on $k_{i}$ now varies the magnitude of $\underline{k}$, and selects out peaks at $r_{j}$ in space for which $\chi(\underline{k})$, through the $F_{j} s$, contains phase factors exp[ikr $r_{j}$. This method has been demonstrated to suppress twin images $[5 b, 15 b]$, most multiple scattering effects [5b], and self-interference effects [18b]. For example, encouraging experimental images have been obtained for bulk Cu(001) by Terminello et al. [14b] and bulk Pt(111) by Petersen et al. [16].

As an illustration of how well images can be improved by this summedenergy approach, we show in Fig. 4 a theoretical simulation of images for the same $c(2 \times 2) \mathrm{s} / \mathrm{Ni}(001)$ system [18b]. The sum was over 13 energies between 862 and $1324 \mathrm{eV}$, with a constant $\Delta \mathrm{k}$ step of $0.3 \AA^{-1}$. The hologram was here calculated over the region fxom $30^{\circ}$ above the surface to normal, which should be the most nearly ideal portion of it, with weak, more isotropic, single scattering being dominant. Even with no scattered-wave corrections, the peak positions are here in excellent agreement with the 
(a) |FT|, XY PLANE, EXPERIMENT:

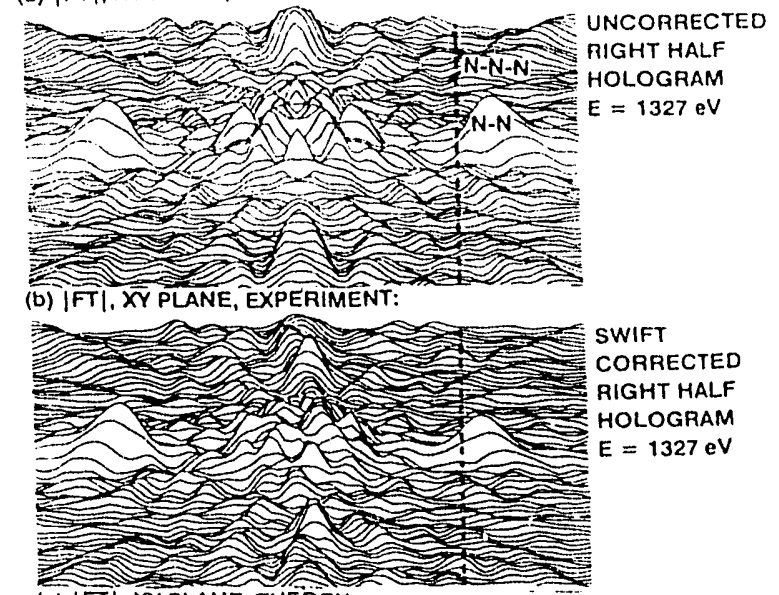

(c) |FT|, XY PLANE, THEORY:

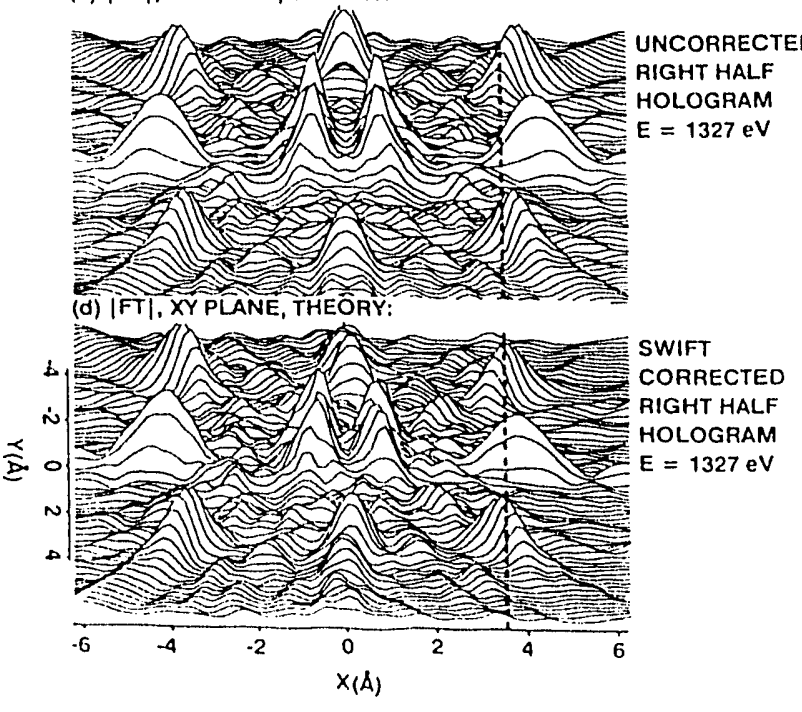

Fig. 3- Fourier transform images in the $s$ plane of $c(2 \times 2) s / N i(001)$ based upon $s$ p emission at 1327 ev. Only the right half of the hologram used in Fig. 2 has been analyzed to minimize real/twin overlap, and the SWIFT scatteredwave correction of Eq. 11 has been used in (b) and (d). Experimental results have been used to derive the images in (a) and (b), and single-scattering theory in (c) and (d). [From Thevuthasan et al., ref. $15 \mathrm{c}$ ]

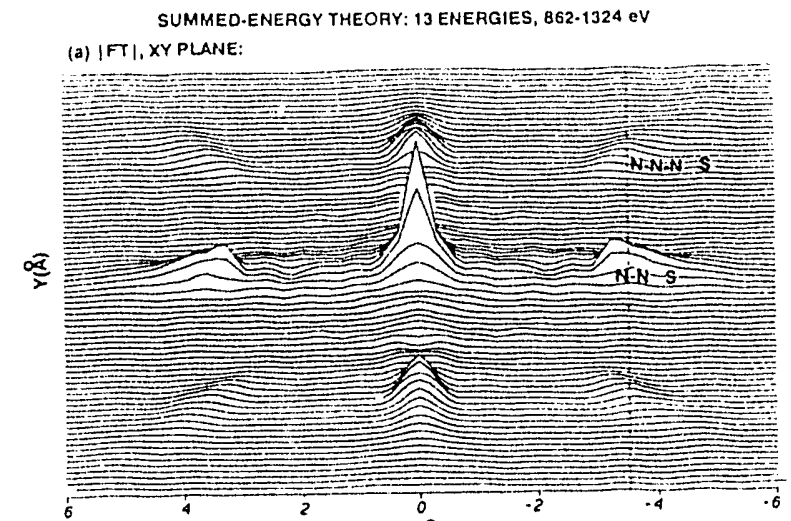

(b) |FT|, XZ PLANE:

$x(\AA)$

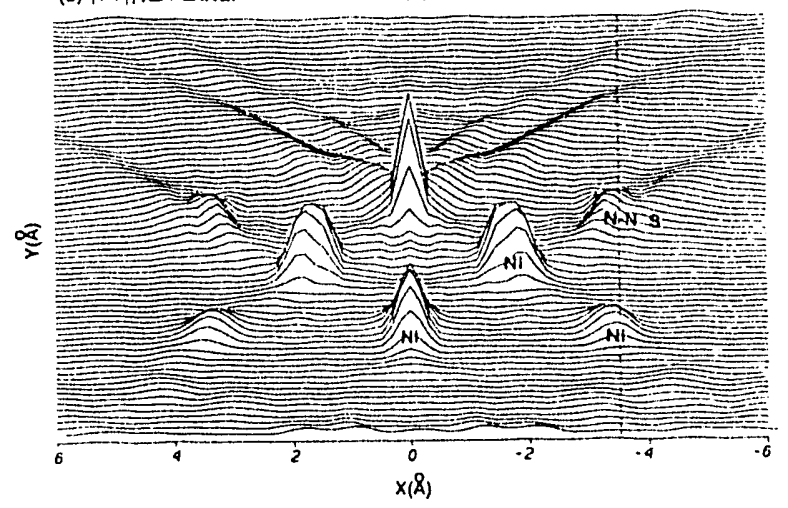

Fig. 4- Theoretical Fourier transform images in the $x y(=s)$ and $x z$ planes based on $s$ p emission from $c(2 \times 2) S / N i(001)$. The hologram spanned the more ideal scattering region from $30^{\circ}$ above the surface to the surface normal. A phased sum of transforms according to Eq. $12 \mathrm{~b}$ has been made over 13 energies between 862 and $1324 \mathrm{eV}$. [From Thevuthasan et al., ref. $18 \mathrm{~b}$ ] 
known structure, the next-nearest-neighbor $s$ atoms are somewhat visible, and the five $\mathrm{Ni}$ atoms underneath a typical s emitter are clearly seen. This simulation makes the use of such summed-energy analyses look extremely encouraging for adsorbate and thin overlayer structure studies.

An important question that immediately arises is how many energies need to be summed over, and how small the steps $\Delta k$ between them need to be to optimally reduce image aberrations and artifacts. Thevuthasan et al. have found in various theoretical simulations that about 10 energies spaced by a constant $\Delta k$ are sufficient to yield essentially complete twin and multiple scattering suppression [15b,18b]. In addition, the size of $\Delta k$ must be small enough to push artifacts (related to aliasing in standard Fouriex transform theory) outside of the range of interatomic distances that are to be studied. The behavior of these artifacts is illustrated in Fig. 5, where images in the $x y$ plane of $c(2 \times 2) s / N i(001)$ are shown for different numbers of energies spanning the the range 862 to $1324 \mathrm{eV}$. Only the right half of the hologram has been analyzed to emphasize the real image due to the nearest-neighbor along $+x$. In going from 1 to 3 to 5 to 7 to 13 energies, we see a gradual suppression of twin-related features in the left half of the image, as expected. But anomalous features remain in circles at multiples of $\pi / \Delta k$ away from the origin and these are fully moved out of the region of interest only in the last panel with 13 energies. Thus, such criteria on the choice of $\Delta k$ are crucial if image artifacts are to be avoided.

Tong and co-workers [23] have also proposed a similar approach for analyzing scanned-energy data so as to simultaneously correct for scattered-wave effects and eliminate twin and multiple-scattering effects. This method does not require data sets over a large solid angle, but rather makes use of several scanned-energy diffraction curves that are then Fourier transformed and used to triangulate on the real-image positions of certain atoms. In effect, what is being done in this procedure is to Fourier transform a $\chi\left(\underline{k}_{q}\right)$ obtained along the direction $k_{q}$ over small steps in $k_{q}$ first and then to carry out a phased sum over several larger steps in direction, as shown below:

$$
u^{\prime \prime \prime}(x, y, z) \propto\left|\Sigma_{q} \exp \left[i \underline{k}_{q} \cdot \underline{r}\right] \int_{\Delta k_{q}} x\left(\underline{k}_{q}\right) \exp \left[-i k_{q} r\right] d k_{q}\right| .
$$

Corrections for scattering amplitudes and/or phase shifts can also be included in this integral, in the same spirit as indicated in Eq. 12 . Encouraging atomic images have been obtained using this approach for ( $\sqrt{3} \times$ $\sqrt{ }$ ) Al on $\mathrm{si}(111$ ) by Wu et al. [17]. Comparison of Eqs. 12a and 13 makes it clear that these two approaches are fundamentally equivalent, in that they just interchange the order of integration and summation with the same overall phase factor of $\exp [-i k r] \exp [\underline{i} \underline{\underline{r}} \underline{\underline{r}}]=\exp [-i k r(1-\cos \theta)]$. However, the first emphasizes finer steps in $\mathbf{k}$ and the other finer steps in $k$. Thus, if both are carried out over equivalent ranges of $\Delta \mathbf{k}_{x^{\prime}} \Delta \mathbf{k}_{y^{\prime}}$ and $\Delta \mathbf{k}_{z^{\prime}}$ one would expect roughly equal resolutions in the coordinates $x, y$, and $z$, provided that the $\underline{k}$ steps are sufficiently small in all directions to avoid spurious features due to the non-cancellation of twin and multiple scattering features (cf. Fig. 5). For a given image accuracy, it is thus expected that these two approaches would require about the same size of data set. Data analyzed using both of these variable-energy approaches has in fact been presented in this symposium [16b and 16c, respectively], and either one absolutely requires the use of synchrotron radiation.

\section{PHOTOELECTRON DIFFRACTION WITH CIRCULARLY-POLARIZED RADIATION}

If instead of linearly-polarized radiation, left or right circularlypolarized radiation is used for excitation, two distinct kinds of circular 
Fig. 5- Theoretical Fourier transform dmages for $c(2 \times 2) s / N i(001)$ in the $s$ plane obtained using only the right half of a hologram extending from $10^{\circ}$ to $50^{\circ}$ above the surface. Data are shown for different numbers of energies in a phased sum according to Eq. 12b, but with no scatteredwave correction: (a) 1 energy, (b) $=3$ energies, $(c)=5,(d)=7$, and $(e)=13$. The multiples of $\pi / \Delta k$ at which artifacts can remain on spherical surfaces surrounding the origin are also indicated; the shaded peaks all occur at such positions. [From Thevuthasan et al., ref. 18b.]
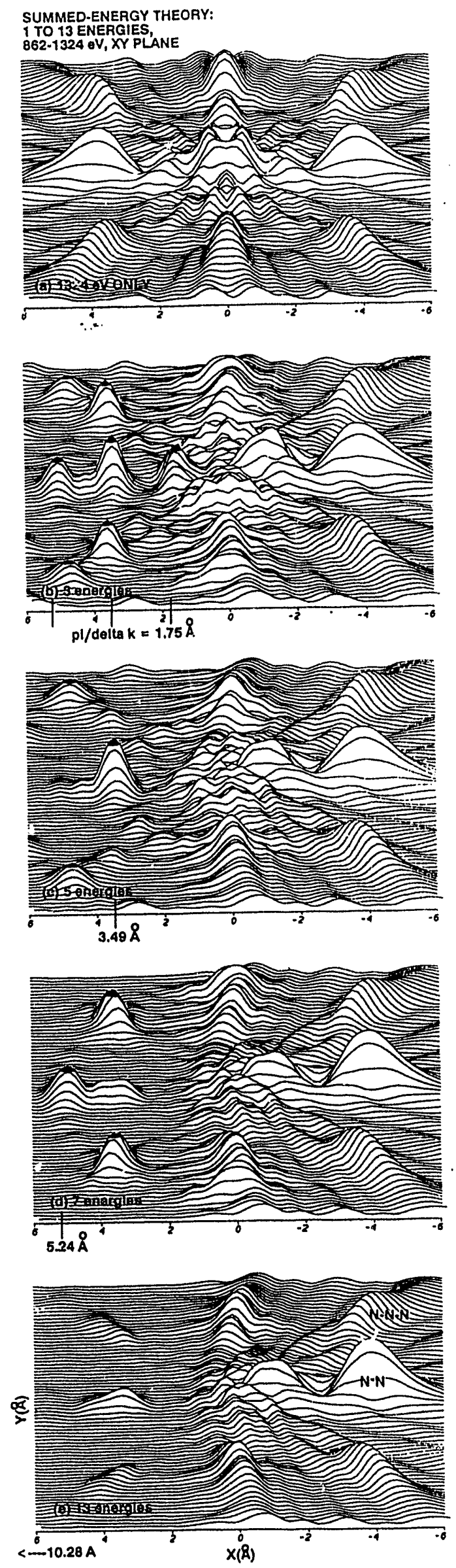
dichroism (CD) can occur: one due to emission in some sort of chiral experimental geometry ("normal" dichroism), and one due to spin-orbit splitting in the presence of an external magnetic field (magnetic $C D$ or MCD). The latter is based on the well-known Fano effect first discussed for atoms: left and right polarized radiation can cause preferential excitation of spin-up or spin-down electrons, even if there were equal populations of the two types in the initial filled core states. In either case, the degree of dichroid asymmetry can be measured as a function of $k$ via

$$
A^{C D}(\underline{k})=\left[I^{R C P}(\underline{k})-I^{L C P}(\underline{k})\right] /\left[I^{R C P}(\underline{k})+I^{L C P}(\underline{k})\right],
$$

where $I^{R C P}$ and $I^{\text {LCP }}$ are the intensities measured with right and left polarized light, respectively. Very few measurements of the $k$ dependence of $A^{C D}$ in core-level emission have been made to date, but we illustrate the types of effects expected with two examples.

Bansmann and co-workers [24a] have studied normal $\mathrm{CD}$ in $\mathrm{C}$ 1s emission from Co adsorbed on Pd(111) in a chiral experimental geometry. Some of their experimental data as a function of electron emission angle $\theta$ is shown in Fig. 6, together with theoretical calculations based on several models. The effects are quite pronounced, being as large as \pm 758 variations in $A C D$. The three theoretical curves all agree reasonably well with the data: two are based upon treating an isolated co molecule only, and one includes the effect of the Pd substrate. Two of these cuxves (-- - and - - - -) have been calculated by Westphal et al. [24b] in a single-scattering diffraction picture of the outgoing wave, thus emphasizing the fact that it is only through photoelectron diffraction that normal circular dichroism can manifest itself in core-level emission. Diffraction theory including the effects of the underlying Pd atoms (- - - ) clearly suggests that the substrate could produce important effects on such data, although there are as yet no conclusive experimental data indicating such effects. The future measurement of circular dichroism in core emission with synchrotron radiation from insertion devices designed to produce high-brightness circularly-polarized radiation, coupled with analysis in terms of more accurate cluster-based multiple-scattering calculations [6d], thus represents a very interesting new direction of study in photoelectron diffraction.

MCD has so far been studied only for a few cases in core-level emission, and then only with a fixed emission direction. In Fig. 7, we show the first data of this type due to Baugarten et al. [25a] for Fe $2 p_{1 / 2,3,2}$ emission from Fe(110). In the lower part of (a) are shown two partial spectra obtained with the sample magnetization parallel to and anti-parallel to the direction of helicity of circularly-polarized radiation; this is equivalent to changing from right to left polarization in the frame of the sample. The full spectrum in (a) represents an average over the two magnetizations. In (b), $A C D$ is plotted, and it is clear that significant effects of the order of a few 8 are seen, even if they are much smaller than those found for normal CD in Fig. 6 similar results have been obtained by Waddill et al. [25b] for Fe 2p emission from thin overlayers of Fe on Cu(001), again for a fixed direction of emission. Both sets of data have been qualitatively explained in terms of preferential excitation of photoelectrons of one spin or another in the $2 p_{1 / 2}$ and $2 p_{3 / 2}$ peaks, combined with a spin-dependent splitting in the core level that is probably linked to multiplet effects. However, no interference between the $l_{f i n a l}=$ 0 and 2 photoelectron channels has yet been included, nor has any spindependent final-state scattering and diffraction been considered. It is aiso ciear that normai CD Cầ comsxist with MCD, and that both types of effect in general need to be considered simultaneously. Thus, future 


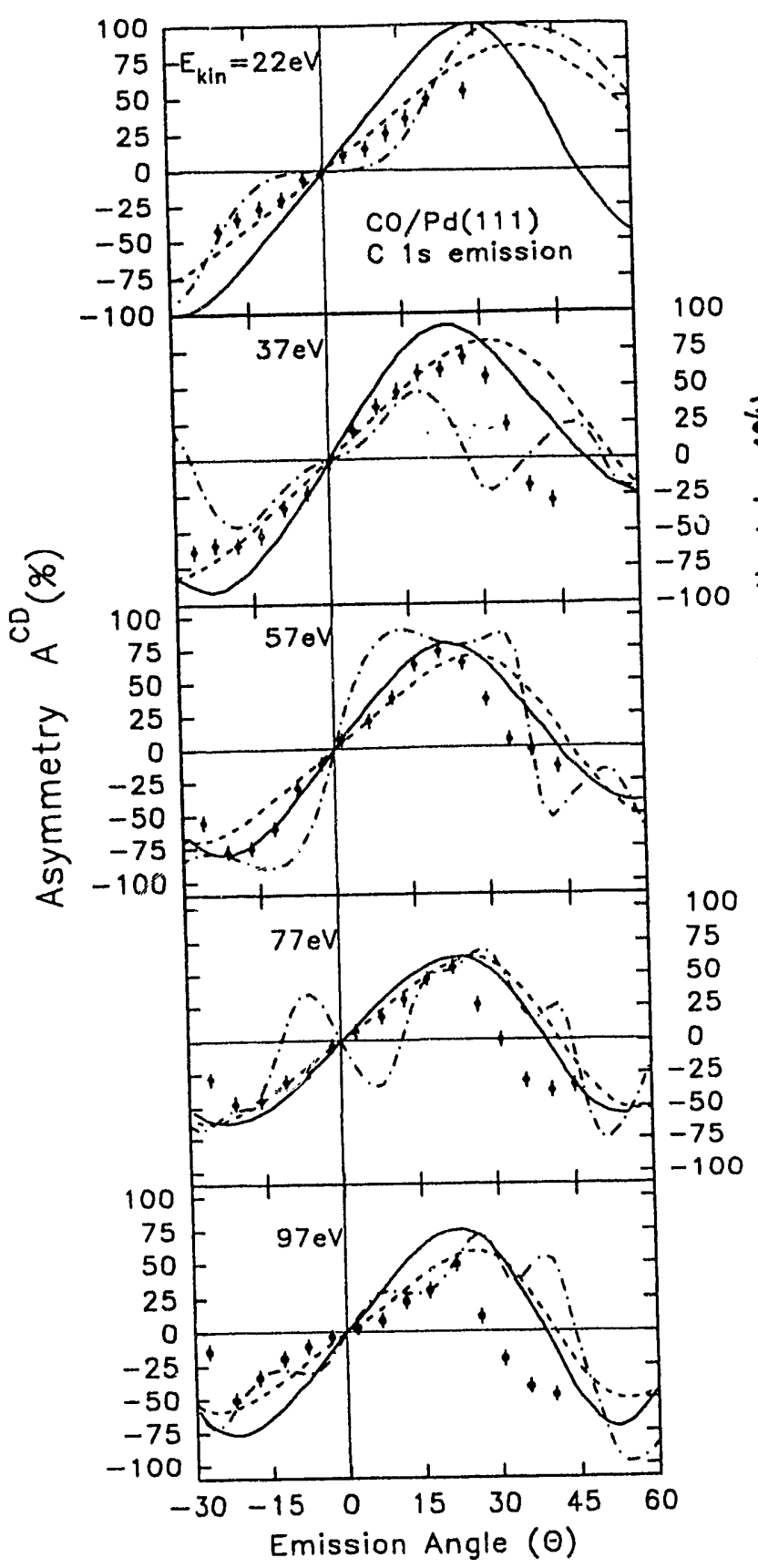

Fig. 6- Normal circular dichroism in $c$ is emission from co adsorbed on $\mathrm{Pd}(111)$. The experimental data and solid theoretical curve are from Bansmann et al. (ref. 24a), and the other two theoretical curves (---- = co only and - - = Co plus 19 Pd substrate atoms, with $\theta$ scan in the $[10,-1]$ azimuth) are from Westphal et al. (ref. 24b) and are based upon single-scattering diffraction calculations.

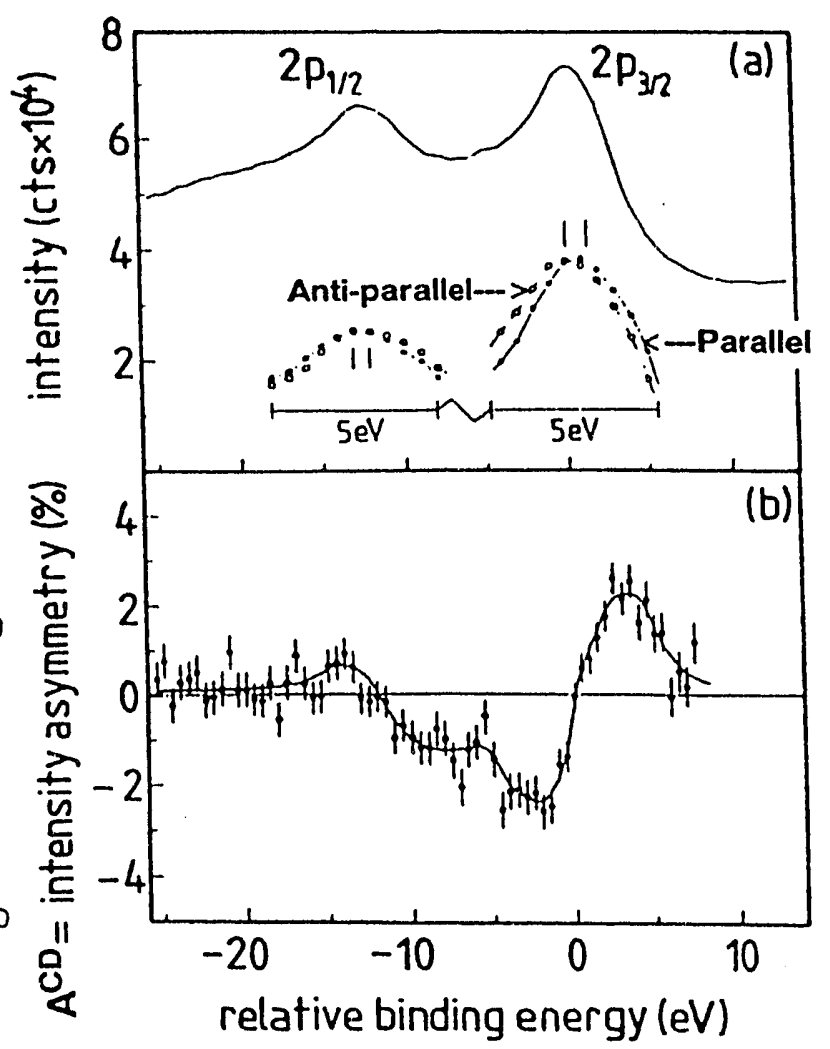

Fig. 7- Magnetic circular dichroism in Fe $2 p$ emission from Fe(110). In (a), the average full spectrum is shown together with data for photon incidence parallel-to and anti-parallel-to the specimen magnetization. In (b), the asymmetry as calculated from Eq. 14 is plotted. [From Baumgarten et al., ref. 25a] 
studies in which both normal $C D$ and $M C D$ are measured over a range of $\underline{k}$ and analyzed more precisely with inclusion of both of these effects represent an area of exciting future development in photoelectron diffraction.

\section{PHOTOELECTRON DIFFRACTION/HOLOGRAPHY WITH SPIN RESOLUTIOH}

If the spin of an outgoing photoelectron can somehow be determined, either through its origin in a well-defined multiplet splitting [10] or through direct external measurement with respect to an external magnetization axis [11], then the spin-dependent aspects of photoelectron diffraction can be studied, for example, by comparing the patterns produced by exiting spin-up and spin-down electrons. These effects were first studied by sinkovic et al. [10] and made use of multiplet-split levels to provide the spin resolution. These experimental data provided evidence for a high-temperature loss of short-range antiferromagnetic order that had not been observed previously. Such spin-dependent photoelectron scattering and diffraction will also clearly be an important part of the analysis of MCD data such as that described in the last section.

In two recent studies the additional possibility of spin-polarixed photoelectron holography has been considered [26]. Although there is as yet no experimental data on this subject, Kaduwela et al. [26a] have carried out model calculations on simple clusters. Some of these results are shown in Fig. 8 for a two-atom cluster in which one $\mathrm{Mn}^{2+}$ ion is the emitter and the other is a magnetically-ordered scatterer. In order to look for spin-dependent exchange effects in the scattering, Fouriertransform images $U(x, y, z)$ were calculated from Eq. 9 for outgoing spin-up and spin-down electrons; no scattered-wave correction was used in order to focus on the spin-dependent differences in the images. The kinetic energy was held constant at $120 \mathrm{eV}$ for both cases. The exchange interaction with the five parallel-coupled 3 d electrons was included in the scattering potential if the photoelectron spin was parallel to the net spin of the $\mathrm{Mn}^{2+}$ scatterer $(\uparrow, \uparrow$ or $\downarrow, \downarrow)$, and was omitted if the photoelectron spin was antiparallel to the scatterer $\operatorname{spin}(\uparrow, \downarrow$ or $\downarrow, \uparrow)$. The two simplest measures of these exchange effects are:

$$
\Delta(x, y, z, \uparrow-\downarrow, \uparrow)=U(x, y, z, \uparrow, \uparrow)-U(x, y, z, \downarrow, \uparrow),
$$

which is simply a difference of two images, and

$$
\Delta^{\prime}(x, y, z, \uparrow-\downarrow, \uparrow)=\left|F_{\sigma}(x, y, z, \uparrow, \uparrow)-F_{\sigma}(x, y, z, \downarrow, \uparrow)\right|,
$$

in which $F$ is the (complex) Fourier transform integral within $U$ and the absolute value is taken after calculating the difference. The second spin argument here is the orientation of the scatterer, here chosen to be up. Through its sign, $\Delta$ can be shown to be sensitive to the orientation of the scatterer, whereas the always-positive $\Delta^{\prime}$ can be shown to measure more directly the strength of the spin-dependent exchange scattering.

In Fig. 8, the two image functions $\Delta$ and $\Delta^{\prime}$ are plotted for the two different orientations of the scatterer: spin-up in parts $(a .2)-(a .4)$ and spin-down in parts (b.2)-(b.4). The effects seen here are 10-15\% of the magnitude of the peaks in the direct $U$ images, and thus should be measurable, especially from higher-quality experimental data obtained with a next-generation synchrotron radiation source. As expected from their definitions, $\Delta$ and $\Delta^{\prime}$ exhibit different behavior on flipping the scatterer spin: $\Delta$ changes in sign, whereas $\Delta^{\prime}$ does not. Thus, it has been suggested that the locations of near-neighbor magnetic scatterers could be determined via $\Delta^{\prime}$, and actual spin flips (e.g., as temperature is changed) could be detected via $\Delta[26]$. 


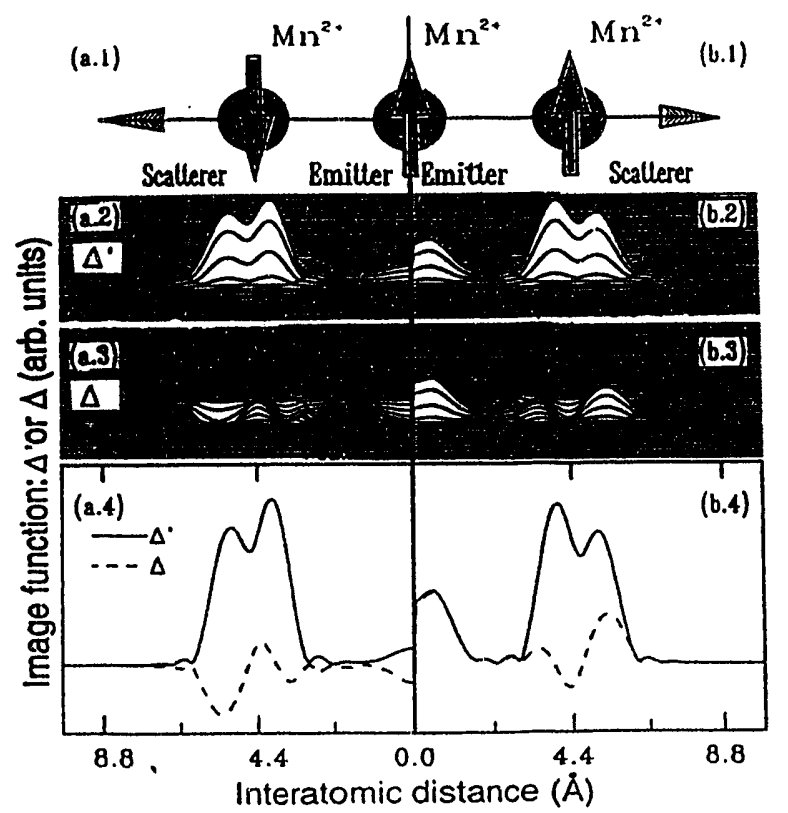

Fig. 8- Holographic (spin-up) (spin-down) difference functions $\Delta$ and $\Delta^{\prime}$ for a cluster of two $\mathrm{Mn}^{2+}$ ions consisting of an emitter and scatterer that are 4.4 A apart. The outgoing electron energy is $120 \mathrm{eV}$ for both spin-up and spindown. (a.1)-(a.4) are for the scatterer spin down. (b.1)-(b.4) are for the scatterer spin down. [From Kaduwela et al., ref. 26a]

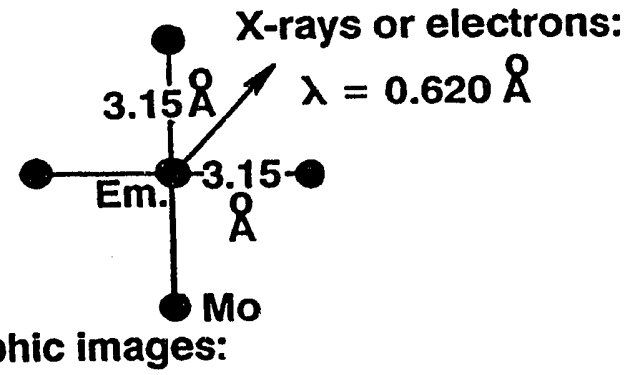

Holographic images:

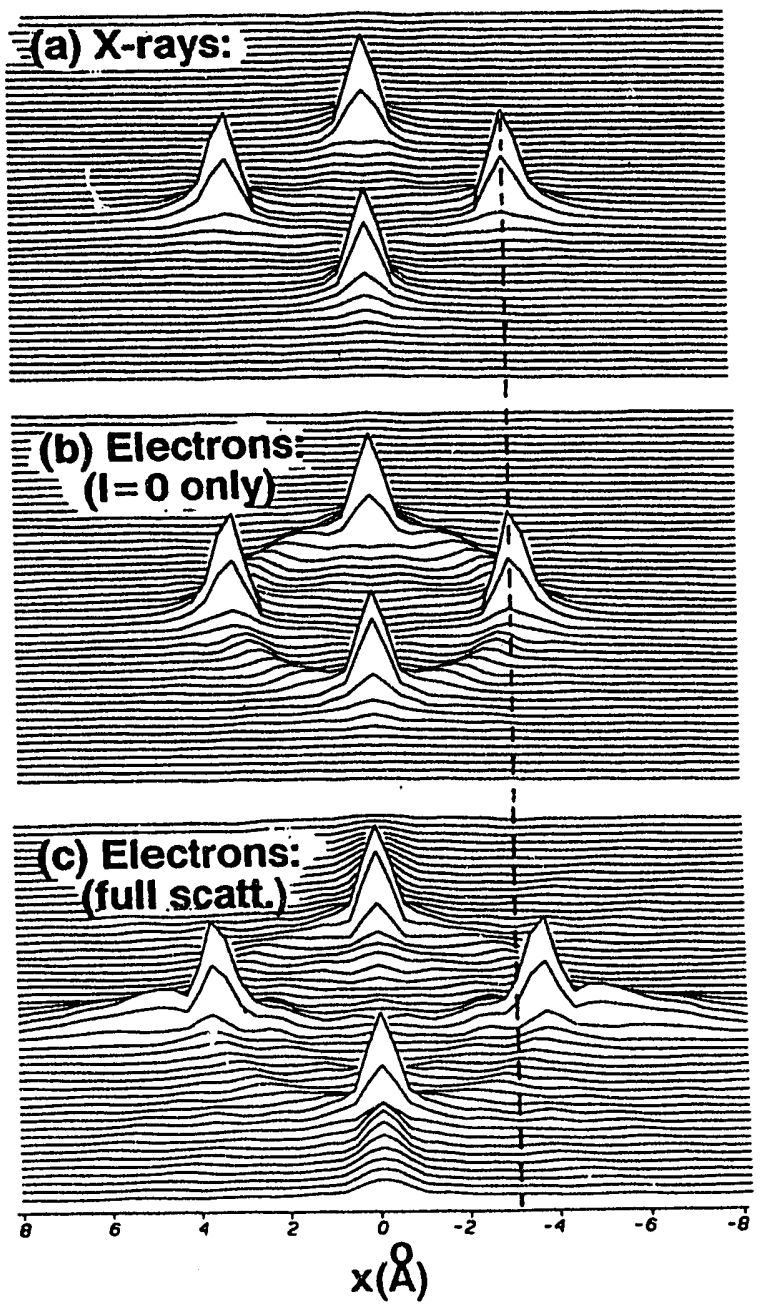

Fig. 9- Fourier transform images for a 5-atom Mo cluster with the emitter in the center are compared for both $x$-ray emission at $20 \mathrm{keV}$ and electron emission at $391 \mathrm{ev}$, with the wavelength equal to 0.620 A for both. (a) $x$-ray produced image. (b) Image produced with artificially weakened electron scattering (s-wave only). (c) Image produced with full-strength electron scattering. [From Len et al., ref. 27$\}$ 


\section{X-RAY FLUORESCENCE HOLOGRAPHY}

Finally, we consider another potentially useful structural probe: the holographic analysis of diffraction patterns in core-level x-ray fluorescence, as first suggested by szöke [4] and recently considered more quantitatively in theoretical calculations by Len et al. [27]. The outgoing fluorescence $x$-ray serves as the reference wave, and the scattered-x-ray components serve as the object waves, just as in photoelectron holography. But the scattering of $x$-ray by atoms is much weaker and more ideal than that of electrons: the scattering amplitude is much more nearly isotropic (although still stronger in the forward direction) and is about 5,000-10,000 times weaker than for an electron at the same wavelength, and the scattering phase shift is for present purposes negligibly small over all scattering angles. Thus, holographic images should be much more accurate, although also much more difficult to measure due to the weaker effects expected.

Fig. 9 shows some results from this study [27] for a 5-atom cluster of Mo atoms, as shown at the top of the figure. Holographic images in the plane of the cluster are shown for emission of both $20 \mathrm{keV} x-r a y s$ and 391 ev electrons from the central atom. The wavelength for both outgoing waves is the same at $0.62 \AA$. It is clear that the $x$-ray image in (a) yields peaks that are much closer to the true scatterer positions than the electron image in (c). Intentionally weakening the electron scattering by including only the more ideal s-wave component of it, as shown in (b), is found to $y$ ield images of similar quality to those of $x$-rays.

Thus, $x$-ray fluorescence holography (XFH) shows promise as a new structural tool that could be applied to both surface and bulk problems due to the greater $x$-ray escape lengths. However, this method will require the measurement of energy-resolved diffraction patterns over a large solid angle and with high statistical accuracy for effects that will be only about $1 / 10^{4}$ of the total signal. In addition, the use of fluoresence $x-$ rays of fixed energy would prevent using a summed-energy approach such as Eq. 12 to suppress twin images. Nonetheless, with high-intensity undulator radiation for excitation and multichannel energy-dispersive detection, it should be possible in the future to at least test this intriguing idea.

\section{REFERENCES}

+ Work supported by the U.S. Department of Energy, Basic Energy Sciences, Materials Science Division, under Contract DOE-AC03-76sF00098, and the office of Naval Research, under Contract No0014-90-J-1457.

[1] (a) C.S. Fadley, Phys. Scripta T17, 39 (1987); (b) in Synchrotron Radiation Research: Advances in Surface Science, edited by R.z. Bachrach (Plenum, New York, 1992); (c) review to appear in Surface Science Reports.

[2] (a) S.A. Chambers, I.M. Vitomirov, S.B. Anderson, H.W. Chen, T.J. Wagner, and J.H. Weaver, superlatt. and Microstruct. $\underline{3}, 563$ (1987); S.A. Chambers, Adv. in Phys. 40, 357 (1990); S.A. Chambers, Surf. Sci. Repts. 16, 261 (1992); (b) W.F. Egelhoff, Jr. in Critical Reviews in solid state and Materials Sciences, 16, 213 (1990).

[3] H.P. Bonzel, review to appear in Prog. in Surf. Sci.

[4] A. Szöke, in short wavelength coherent Radiation: Generation and Applications, edited by D.T. Attwood and $J$. Boker, AIp Conference Proceedings No. 147 (AIP, New York, 1986). 
[5] (a) J.J. Barton, Phys. Rev. Lett. 61, 1356 (1988); (b) J.J. Barton, J. Electron Spectrosc. $\underline{51}, 37$ (1990).

[6] (a) C.H. $L i$ and S.Y. Tong, Phys, Rev. Lett. 42,901 (1979); (b) J.J. Barton and D.A. Shirley, Phys. Rev. B32, 1892 (1985); and Pbys. Rev. B32, 1906 (1985); (c) A.P. Kaduwela, G.S. Berman, D.J. Friedman and C.S. Fadley, Phys. Scripta 41, 948 (1990); (d) A.P. Kaduwela, D.J. Friedman, and C.S. Fadley, J. Electron Spectrosc. 57, 223 (1991).

[7] D.J. Friedman and C.S. Fadley, J. Electron Spectrosc. 51, 689 (1990).

[8] P.J. Orders and C.S. Fadley, Phys. Rev. B27, 781 (1983); M. Sagurton, E.I. Bullock, and C.s. Fadley, Phys. Rev. $\underline{30}, 7332$ (1984).

[9] (a) D. Sebilleau, M.C. Desjonqueres, D. Chaveau, C. Guillot, J. Lecante, G. Treglia, and D. Spanjaard, Surf. Sci. Lett. 185, L527 (1987); (b) A. Nilsson, H. Tillborg, and N. Mårtensson, Phys. Rev. Lett. 67, 1015 (1991); (c) K.U. Weiss et al., Phys. Rev. Lett. 69, 3196 (1992); (d) J.D. Denlinger, E. Rotenberg, U. Hessinger, M. Leskovar, and M.A. Olmstead, J. Vac. Sci. Tech., to appear.

[10] B. Sinkovic, B.D. Hermsmeier, and C.s. Fadley, Phys. Rev. Lett. 55, 1227 (1985); B. Sinkovic, D.J. Friedman, and C.s. Fadley, J. Magn. Magn. Mater. 92, 301 (1991); B.D. Hermsmeier, J. Osterwalder, D.J. Friedman, and C.s. Fadley, Phys. Rev. Lett. 62, 478 (1989); B.D. Hermsmeier, J. Osterwalder, D.J. Friedman, B. Sinkovic, T.T. Tran, and C.S. Fadley, Phys. Rev. B42, 11895 (1990).

[11] F.U. Hillebrecht, R. Jungblut, and E. Kisker, Phys. Rev. Lett. 65, 2450 (1990).

[12] I.J. Terminello, X.S. Zhang, Z.Q. Huang, S. Kim, A.E. Schach von Wittenau, K.T. Leung, and D.A. Shirley, Phys. Rev. B 38, 3879 (1988); L. -Q. Wang, Z. Hussain, Z.Q. Huang, A.E. Schach von Wittenau, D.A. Shirley, and D.W. Lindle, Phys. Rev. B444, 13771 (1991).

[13] V. Fritzsche and D.P. Woodruff, Phys. Rev. B46, 16128 (11992).

[14] (a) G.R. Harp, D.K. Saldin, and B.P. Tonner, Phys. Rev. Lett. 65,1012 (1990); G.R. Harp, D.K. Saldin, and B.P. Tonner, Phys. Rev. B42, 9199 (1990); (b) L.J. Terminello, J.J. Barton, and D.A. Lapiano-Smith, J. Vac. Sci. Technol. B10, 2088 (1992) and private communication.

[15] (a) G.S. Herman, S. Thevulhasan, T.T. Tran, Y.J, Kim, and C.S. Fadley, Phys. Rev. Lett. 68,650 (1992); (b) S. Thevuthasan, G.S. Herman, A.P. Kaduwela, T.T. Tran, Y.J. Kim, R.S. Saiki, and C.S. Fadley, J. Vac. Sci. Technol. A10, 2261 (1992); (C) S. Thevuthasan, R.X. Ynzunza, E.D. Tober, C.S. Fadley, A.P. Kaduwela, and M.A. van Hove, PhY. Rev. Lett. 70,595 (1993)

[16] (a) Y. Zhou, X. Chen, J.C. Campuzano, D.K. Saldin, and H. Ding, these proceedings; (b) B. Petersen, I. Terminello, J.J. Barton, and D.A. Skirley, these proceedings; (C) G.D. Waddill and J.G. Tobin, to be published.

[17] H. Wu, G.J. Lapeyre, H. Huang, and S.Y. Tong, to be published.

[18] (a) S. Thevuthasan, G.S. Herman, A.P. Kaduwela, R.S. Saiki, Y.J. Kim, W. Niemczura, M. Burger and C.S. Fadley, Phys. Rev. Lett. 67,469 (1991) (b) S. Theruthasan, P.M. Len, and C.S. Fadiey, to be published. 
[19] G.R. Harp, D.K. Saldin, Z. Chen, Z.L. Han, and B.P. Tonner, J. Electron spectrosc. 258, 313 (1991).

[20] (a) B.P. Tonner, Z.-I. Han, G.R. Harp, and D.K. Saldin, Phys. Rev. B43, 14423 (1991); D.K. Eaidin, G.R. Haxp, and B.P. Tonner, Phys. Rev. B45, 9629 (1992); (b) S.Y. Tong, C.M. Wei, T.C. ZhaO, H. Huang, and H. Li, PhYs. Rev. Lett. 66, 60 (1991).

[21] D.K. Saldin, G.R. Harp, B.I. Chen and B.P. Tonner, Phys. Rev. B44, 2480 (1992).

[22] R.S. Saiki, A.P. Kaduwela, J. Osterwalder, D.J. Friedman, C.S. Fadley, and C.R. Brundle, Surf. Sci. 279, 305 (1992).

[23] S.Y. Tong, H. Li, and H. Huang, Phys. Rev. Lett. 67, 3102 (1992); S.Y. Tong, H. Huang, and C.M. Wei, Phys. Rev. B46, 2452 (1992).

[24] (a) J. Bansmann, Ch. Ostertag, G. Schönhense, F. Fegel, C. Westphal, M. Getzlaff, F. Schafers, and H. Petersen, Phys. Rev. B46, 13496 (1992); (b) A.P. Raduwela, C. Westphal, M. Van Hove, and C.s. Fadley, to be published..

[25] (a) G. Baumgarten, C.M. Schneider, H. Petersen, F. Schafers, and J. Kirschner, Phys. Rev. Lett. 65, 492 (1990); (b) G.D. Waddill, J. Tobin, and D.R. Pappas, Phys. Rev. B46, (1992).

[26] (a) A.P. Kaduwela, Z. Wang, M.A. Van Hove, and C.S. Fadley, to be published; (b) E.M.E. Timmermans, G.T. Trammell, and J.P. Hannon, to be published.

[27] P.M. Len, S. Thevuthasan, C.S. Fadley, A.P. Kaduwela, and M.A. Van Hove, to be published. 

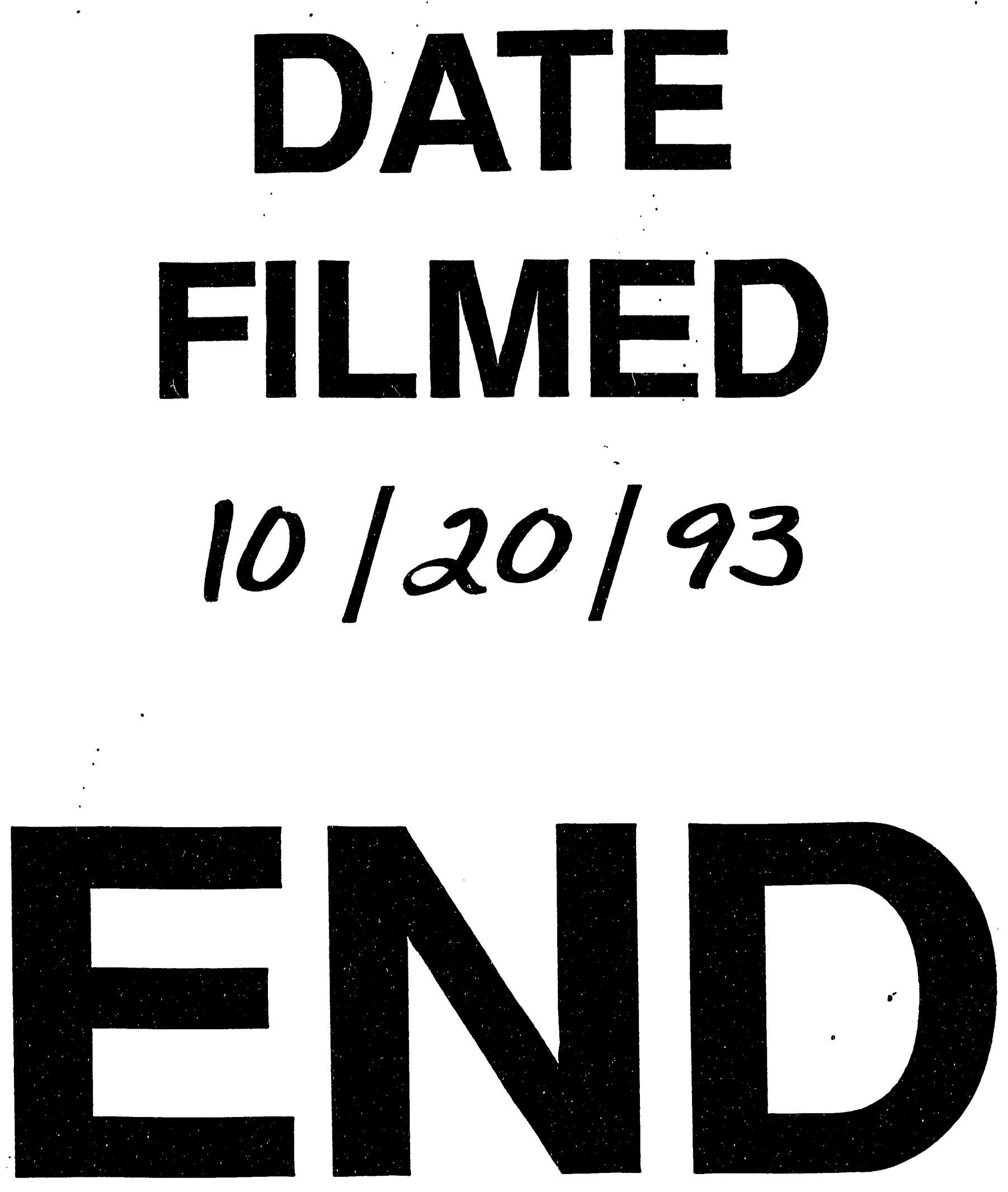
